\title{
Neuropeptide-S (NPS) Receptor Genotype Modulates Basolateral Amygdala Responsiveness to Aversive Stimuli
}

\author{
Udo Dannlowski*, 1,4, Harald Kugel 2,4, Friederike Franke', Anja Stuhrmann', Christa Hohoff', \\ Peter Zwanzger', Thomas Lenzen', Dominik Grotegerd', Thomas Suslow', ${ }^{1,3}$, Volker Arolt', \\ Walter Heindel ${ }^{2}$ and Katharina Domschke' \\ 'Department of Psychiatry, University of Münster, Münster, Germany; ${ }^{2}$ Department of Clinical Radiology, University of Münster, Münster, \\ Germany; ${ }^{3}$ Department of Psychosomatic Medicine and Psychotherapy, University of Leipzig, Leipzig, Germany
}

\begin{abstract}
Recent studies point to a role of neuropeptide-S (NPS) in the etiology of anxiety disorders. In animal models, NPS and its receptor (NPSR) were shown to be highly expressed in the amygdala, a central structure in the fear circuit, also known to be hyper-responsive in anxiety disorders. Recently, a functional polymorphism in the NPSR gene (rs32498I A/T) has been associated with panic disorder and anxiety sensitivity. However, the role of NPSR gene variation in the modulation of fear-related amygdala responsiveness remains to be clarified. In 79 healthy subjects genotyped for NPSR rs324981, amygdala responses were assessed by means of fMRI. The participants were presented with fear-relevant faces in a robust emotion-processing paradigm frequently used to study amygdala responsiveness. We observed a strong association of NPSR T-alleles with right amygdala responsiveness to fear-relevant faces. The association peak was located in the BLA. Furthermore, responsiveness to aversive stimuli within this BLA cluster predicted a participant's self-reported harm avoidance but not depression level. We conclude that NPSR genotype is associated with increased amygdala responsiveness to fear-relevant stimuli. Thereby, NPSR rs32498I apparently causes an indirect effect on anxiety-related traits and potentially contributes to the pathogenesis of anxiety disorders by shaping fear-related limbic activity.

Neuropsychopharmacology (20II) 36, 1879-1885; doi:10.1038/npp.201।.73; published online 27 April 201।
\end{abstract}

Keywords: neuropeptide-S; emotion; amygdala; anxiety; fMRI; imaging genetics

\section{INTRODUCTION}

Anxiety disorders are highly prevalent and debilitating psychiatric diseases with a moderate to high degree of heritability (Hettema et al, 2001). Understanding the underlying molecular genetic pathomechanisms and their associated neurobiological abnormalities is a major goal of current research efforts. Neuropeptides have been suggested to have a crucial role in the pathogenesis of stress, arousal, and anxiety. Particularly, the recently discovered neuropeptide-S (NPS) and agonists at its receptor (NPSR) were reported to elicit anxiolytic effects in several studies using rodent models (Xu et al, 2004; Rizzi et al, 2008; Leonard et al, 2008; Vitale et al, 2008), and thus constitute promising candidates for research on fear processing, anxiety, and anxiety disorders (Reinscheid et al, 2005a; Okamura and Reinscheid, 2007; Pape et al, 2010).

\footnotetext{
*Correspondence: Dr $\cup$ Dannlowski, Department of Psychiatry, University of Munster, Albert-Schweitzer-Strasse II, Munster 48I49, Germany, Tel: + 49 25। 835 660।, Fax: + 49 25। 835 66।2,

E-mail: dannlow@uni-muenster.de

${ }^{4}$ These authors contributed equally to this work

Received 25 February 2011; revised 21 March 2011; accepted 31 March 201I
}

Whereas NPSR precursor mRNA is strongly expressed in the locus coeruleus as the major source of noradrenergic transmission in the brain, NPSR mRNA is widely expressed throughout the central nervous system, including the amygdaloid complex, as investigated in rodent models (cf. Reinscheid et al, 2005a). For example, Jüngling et al (2008) described a cell-specific localization of NPSR expression in the projection neurons of the amygdala, particularly in the basolateral amygdala (BLA) principal neurons. Furthermore, BLA activity was reported to be dependent on a NPS-responsive circuitry (Meis et al, 2008). Consequently, intra-amygdala injections of NPS were shown to modulate fear-potentiated startle (Fendt et al, 2010, lateral amygdala) and anxiety-related behavior (Jüngling et al, 2008, BLA). The BLA is the primary input site of the amygdaloid complex and receives wide projections from thalamic nuclei and sensory association cortices. Whereas the central nucleus seems to be essential for the basic species-specific defensive responses associated with fear, the BLA is apparently associated with encoding the threat value of a stimulus (Davis and Whalen, 2001). Taken together, the NPS system appears to mediate specific effects on synaptic transmission to and within the BLA, which is important for processing of fear (Pape et al, 2010).

In the NPSR gene, a single-nucleotide polymorphism has recently been discovered ( $\mathrm{rs} 324981 \mathrm{~A} / \mathrm{T})$ that leads to 
Table I Task Performance, Sociodemographic, and Affective Characteristics of Study Participants Dependent on NPSR rs32498I A/T Genotype

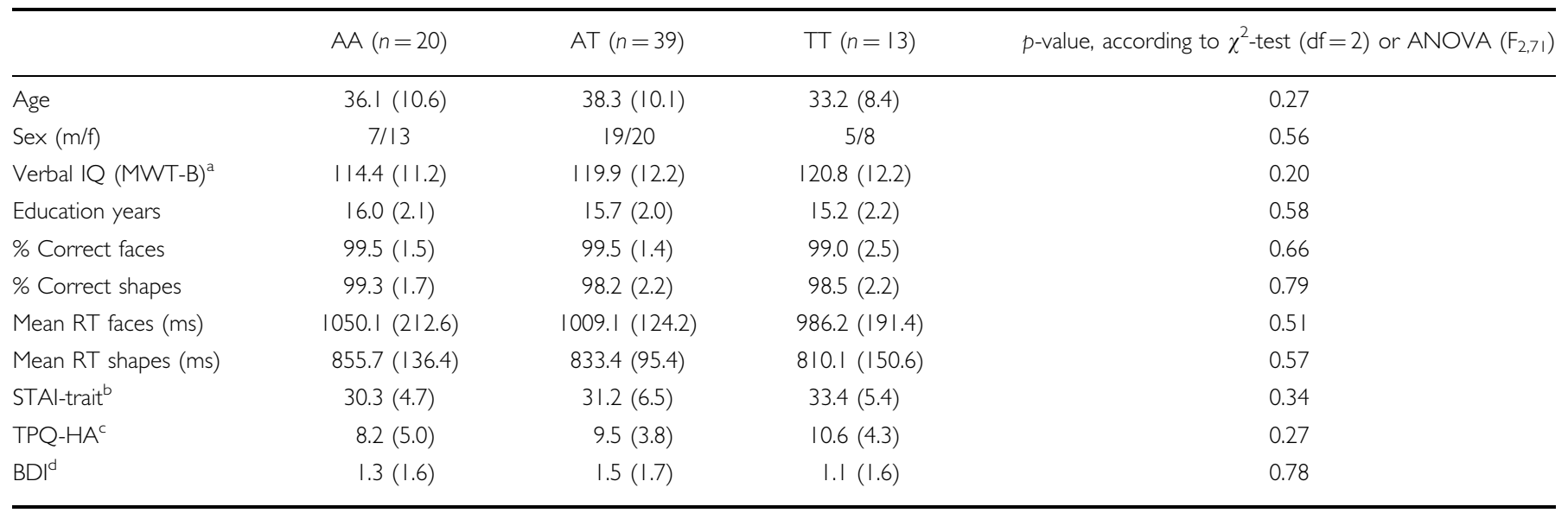

aMWT-B, Mehrfachwahl-Wortschatz-Intelligenztest (Lehrl, 1995)

'STAI-trait, State-Trait Anxiety Inventory (Laux et al, 198I).

'TPQ-HA, Tridimensional Personality Questionnaire, harm avoidance scale (Cloninger, 1987).

'BDI, Beck Depression Inventory (Beck and Steer, 1987).

an Asn/Ile exchange at position 107 (Asn $\left.{ }^{107} \mathrm{Ile}\right)$, with the T-allele $\left({ }^{107} \mathrm{Ile}\right)$ increasing NPSR expression and NPS efficacy at NPSR about 10-fold (Reinscheid et al, 2005b; Bernier et al, 2006). In two previous studies, the NPSR T-allele has consistently been found to be associated with panic disorder, elevated anxiety sensitivity, and increased autonomic arousal (Okamura et al, 2007; Domschke et al, 2010b).

The neurobiological underpinnings of anxiety reaction in healthy subjects and anxiety disorders point to a pivotal role of the amygdala, and amygdala hyper-excitability is regarded as the main neural substrate in the etiology of these illnesses, as an overwhelming majority of neuroimaging studies reported that patients suffering from various anxiety disorders show potentiated amygdala responsiveness to aversive, fear-relevant stimuli (Etkin and Wager, 2007; Sehlmeyer et al, 2009; Domschke and Dannlowski, 2010). Also in healthy controls, several studies reported associations between amygdala responsiveness and (non-pathological) anxiety-related traits (Fakra et al, 2009; Sehlmeyer et al, 2010; Baeken et al, 2010), particularly in the basolateral parts of the amygdala (Etkin et al, 2004).

Given the recent evidence regarding NPS in the etiology of anxiety disorders in humans, and the modulation of (basolateral) amygdala responsiveness already shown in animal models, we sought to investigate whether NPSR rs324981 A/T also impacts human amygdala responsiveness to anxietyrelevant (fearful and angry) facial expressions as measured by functional magnetic resonance imaging (fMRI). We hypothesized that the risk $(\mathrm{T})$ allele is associated with increased amygdala responsiveness to fear-relevant stimuli (angry and fearful faces) presented in a robust paradigm, already used in several previous imaging genetics studies.

\section{MATERIALS AND METHODS}

\section{Subjects}

Seventy-nine right-handed healthy subjects of European ancestry participated in the present study as part of an ongoing project investigating the neurogenetics of emotion processing. All subjects were free from any life-time history of psychiatric disorders according to the DSM-IV criteria (American Psychiatric Association, 1994), diagnosed with the SCID-I interview (Wittchen et al, 1997). Further exclusion criteria were any neurological abnormalities, history of seizures, head trauma or unconsciousness, intake of any psychotropic medication, and the usual MRI contraindications. One male subject had to be excluded for anatomical abnormalities discovered in the structural MRI images (hydrocephalus). Six subjects were excluded for excessive head movement $\left(>2 \mathrm{~mm}\right.$ or $\left.>2^{\circ}\right)$. The TPQ harm avoidance scale was administered (Cloninger, 1987) and trait anxiety was assessed using the State-Trait Anxiety Inventory (STAI, trait version) (Laux et al, 1981). The Beck Depression Inventory (BDI) (Beck and Steer, 1987) was used to measure current depression level. Table 1 lists the sociodemographic and questionnaire data of the study participants dependent on the NPSR rs324981 genotype group. The study was approved by the Ethics Committee of the University of Münster. After a complete description of the study to the participants, written informed consent was obtained. The participants received a financial compensation of $30 €$.

\section{Genotyping}

Participants were genotyped for the functional NPSR rs324981 A/T (Asn ${ }^{107} \mathrm{Ile}$ ) polymorphism according to published protocols (Domschke et al, 2010b). DNA isolated from venous blood samples was amplified by PCR using the primers F: $5^{\prime}$-GAAGGAAAAAAATTAAAAATGAA CCTCCCCAGGATTTCAT and R: 5'-TTCTACCCAGGAGAA AGCGGGCAGTTTGATGCA, resulting in an amplicon size of $353 \mathrm{bp}$. Standard PCR was performed in a $20-\mu \mathrm{l}$ volume containing $45-60 \mathrm{ng}$ of genomic DNA, $10 \mathrm{pmol}$ of each primer, $200 \mathrm{mM}$ dNTPs, $0.4 \mathrm{U}$ Taq DNA Polymerase (Eppendorf, Hamburg, Germany), $50 \mathrm{mM} \mathrm{KCl}, 1.5 \mathrm{mM}$ $\mathrm{MgCl}_{2}$, and $10 \mathrm{mM}$ Tris- $\mathrm{HCl}(\mathrm{pH}$ 8.4). After a 5-min 
denaturation, 35 cycles were conducted consisting of $30 \mathrm{~s}$ at $94{ }^{\circ} \mathrm{C}, 30 \mathrm{~s}$ at $66^{\circ} \mathrm{C}$, and $60 \mathrm{~s}$ at $72^{\circ} \mathrm{C}$, followed by a final extension step of $10 \mathrm{~min}$ at $72{ }^{\circ} \mathrm{C}$. The amplicons were digested with TasI (Fermentas, St Leon-Rot, Germany) (1 U), separated for $2 \mathrm{~h}$ on $15 \%$ polyacrylamide gel, and visualized by silver staining. The Hardy-Weinberg criterion, as calculated by using the online program DeFinetti (http://ihg.gsf.de/cgi-bin/hw/hwa1.pl; Wienker TF and Strom TM), was fulfilled (exact test; $p=0.48$ ).

\section{fMRI Methods}

The experimental fMRI paradigm was a frequently used, robust paradigm for eliciting amygdala responsiveness, which has been used in several previous imaging genetics studies (Hariri et al, 2002, 2005; Pezawas et al, 2005; MeyerLindenberg et al, 2006; Zhou et al, 2008; Fakra et al, 2009). The paradigm consisted of four blocks of a face-processing task alternating with five blocks of a sensorimotor control task. During the face-processing task, participants viewed a trio of faces (all expressing either anger or fear) from the Ekman and Friesen (Ekman and Friesen, 1976) stimulus set and selected 1 of 2 faces (bottom) that was identical to the target face (top). Each face-processing block consisted of six images, balanced for target gender and emotion (angry or fearful). During the sensorimotor control blocks, the participants viewed a trio of geometric shapes (circles and ellipses) and selected 1 of 2 shapes (bottom) that was identical to the target shape (top). Each sensorimotor control block consisted of six different shape trios. All blocks were preceded by an instruction ('Match faces' or 'Match shapes' in German) that lasted $2 \mathrm{~s}$. In the faceprocessing blocks, each of the six face trios was presented for $4 \mathrm{~s}$ with a variable inter-stimulus interval of 2-6 s (mean, $4 \mathrm{~s}$ ), for a total block length of $48 \mathrm{~s}$. In the sensorimotor control blocks, each of the six shape trios was presented for $4 \mathrm{~s}$ with a fixed inter-stimulus interval of $2 \mathrm{~s}$, for a total block length of $36 \mathrm{~s}$. The total task time was $390 \mathrm{~s}$. Participant performance (accuracy and reaction time) was recorded.

The participants held a fiber-optic response pad with two buttons in their right hand. Images were projected to the rear end of the scanner (Sharp XG-PC10XE with additional $\mathrm{HF}$ shielding). $\mathrm{T} 2{ }^{*}$ functional data were acquired using a $3 \mathrm{~T}$ scanner (Gyroscan Intera 3T, Philips Medical Systems, Best, $\mathrm{NL}$ ), using a single-shot echoplanar sequence, with parameters selected to minimize distortion in the region of central interest, while retaining adequate a signal-to-noise ratio $(\mathrm{S} / \mathrm{N})$ and $\mathrm{T} 2^{*}$ sensitivity. Volumes consisting of 34 slices were acquired (matrix $64^{2}$, resolution $3.6 \times 3.6 \times$ $3.6 \mathrm{~mm} ; \mathrm{TR}=2.1 \mathrm{~s}, \mathrm{TE}=30 \mathrm{~ms}, \mathrm{FA}=90^{\circ}$ ). The slices were tilted $25^{\circ}$ from the AC/PC line in order to minimize dropout artifacts in the orbitofrontal and mediotemporal region.

The functional imaging data were realigned and unwarped, spatially normalized to standard MNI space (Montreal Neurological Institute), and smoothed (Gaussian kernel, 6 mm FWHM) using Statistical Parametric Mapping (SPM8; http://www.fil.ion.ucl.ac.uk/spm). Six subjects had to be excluded due to excessive head movement (exclusion criterion $>2 \mathrm{~mm}$ and/or $2^{\circ}$ ). The onsets and durations of the two experimental conditions (faces and shapes) were modeled using a canonical hemodynamic response function in the context of the general linear model (GLM) and the model was corrected for serial correlations. For each participant, one contrast image was generated in each individual fixed-effects first-level analysis comparing activation in response to fear-relevant faces with the shapes baseline. The resulting contrast images were then entered into secondlevel random-effects group analyses.

We first analyzed whether the amygdalae were significantly activated by the task. Therefore, we used a smallvolume correction approach with a threshold of $p<0.05$, family-wise error (FWE)-corrected for the amygdalae. The amygdala was defined according to Tzourio-Mazoyer et al (2002) and the amygdala mask was created by means of the WFU PickAtlas (Maldjian et al, 2003). In a second step, we tested our main hypothesis of amygdala modulation by NPSR genotype by regressing the number of risk (T) alleles on amygdala responsiveness to fear-relevant facial expressions using the same anatomical mask and statistical threshold.

Finally, mean contrast values from the cluster that was significantly modulated by genotype were extracted for each participant and further processed using SPSS 15.0. We correlated these extracted amygdala activation values with trait anxiety and harm avoidance scores, performed posthoc comparisons of the three genotype groups, and tested for gender effects.

For exploratory reasons, a supplementary whole-brain analysis of the effects of NPSR rs324981 T-allele on brain responsiveness was conducted at $p<0.001$, uncorrected, with a cluster threshold of $k=20$ voxels (Table 2 for results). The anatomical labeling for the whole-brain data was performed by using the AAL-Toolbox (TzourioMazoyer et al, 2002), and the Brodmann areas (BAs) were identified by using the Talairach Daemon atlas (Lancaster et al, 2000).

\section{RESULTS}

\section{Behavioral Results}

The genotype groups did not differ regarding behavioral data, any sociodemographic variables, or questionnaire measures (see Table 1).

\section{fMRI Results}

As in previous imaging genetics studies, the paradigm robustly activated the bilateral amygdala (right: $x=28$, $y=-2, z=-24, Z>8, p_{\text {(FWE-corrected) }}<0.001$, cluster size $k=178$; left: $x=-30, y=0, z=-24, Z=7.12, \quad p_{\text {(FWE- }}$ corrected) $<0.001$, cluster size $k=114$ ).

The regression analysis showed a strong positive association of NPSR rs324981 T-alleles and amygdala responsiveness to fearful/angry faces $(x=36, y=-2, \quad z=-24$; $Z=3.91, \quad p_{\text {(uncorrected) }}=0.00004 ; \quad p_{(\text {FWE-corrected })}=0.01$; $r=0.44$, cluster size $k=6$ (Figure 1)). To determine the amygdala sub-region, the SPM Anatomy toolbox version 1.5 (Eickhoff et al, 2005) was used. According to the implemented probabilistic cytoarchitectonic maps (Amunts et al, 2005), the cluster was located in the BLA. Mean contrast values for this cluster were extracted for each subject and further processed using SPSS 15.0 in order to 
Table 2 Results of a Whole-Brain Regression Analysis of NPSR rs32498I T-Alleles on Neural Responsiveness to Fear-Related Facial Expressions Conducted at $p<0.00 \mathrm{I}$, Uncorrected, $k=20$ Voxels

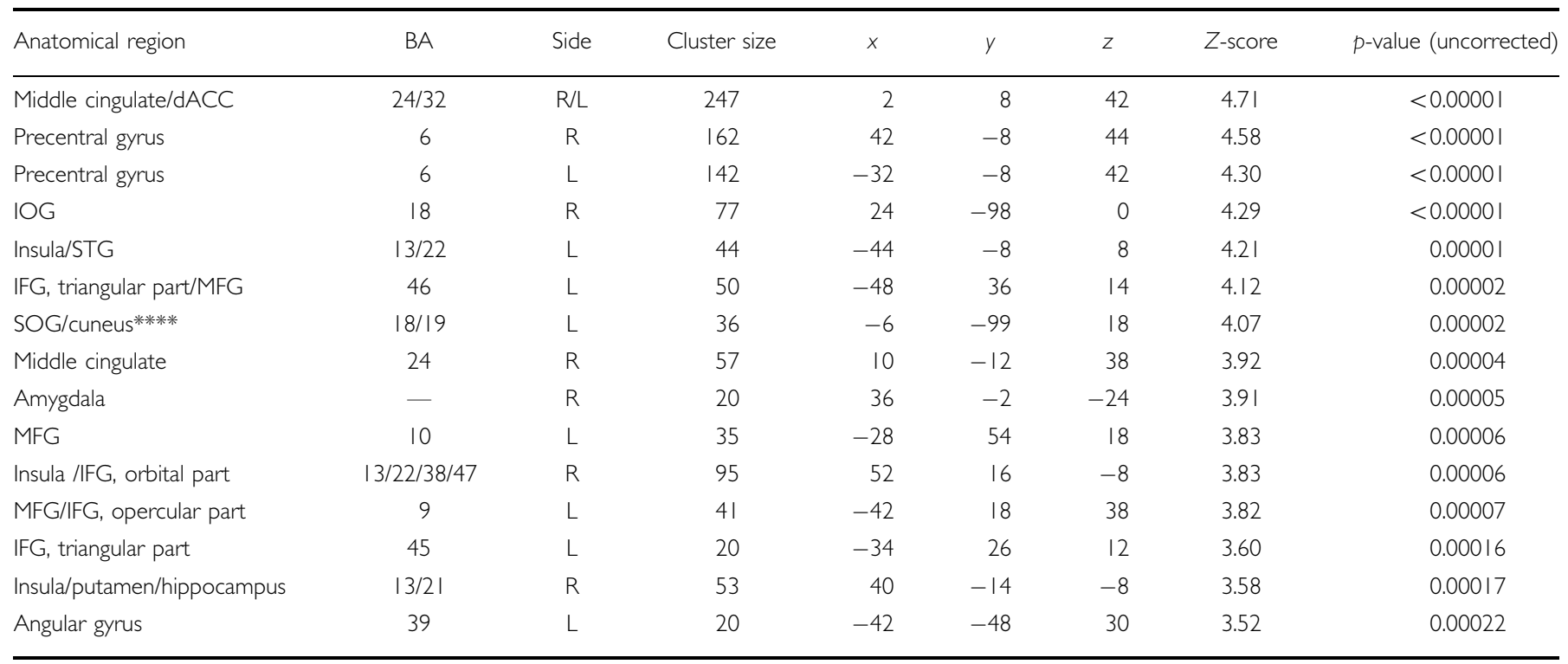

Abbreviations: BA, Brodmann area; IFG, inferior frontal gyrus; IOG, inferior occipital gyrus; MFG, Middle frontal gyrus; SFG, superior frontal gyrus; SOG, superior occipital gyrus.

Coordinates are given in MNI space.
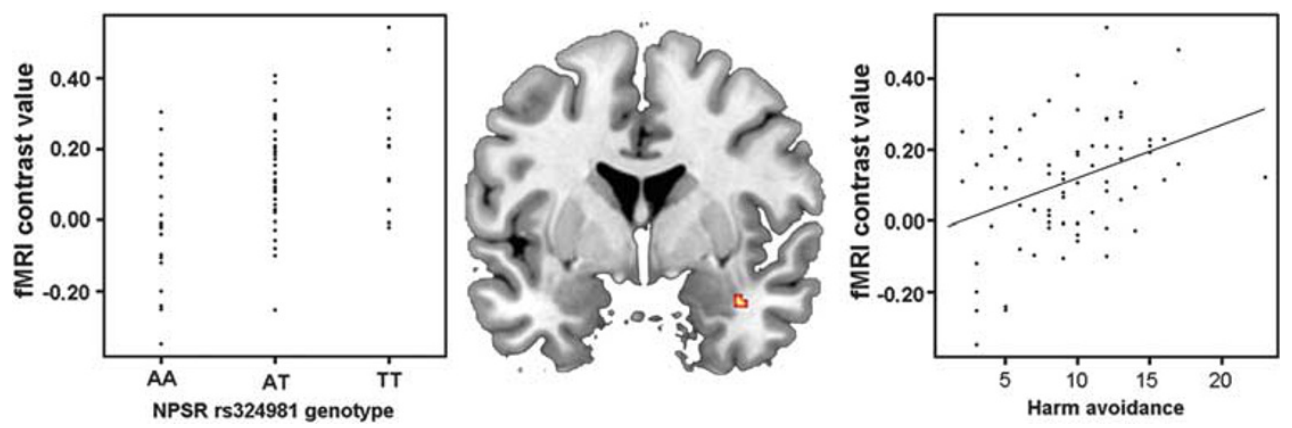

Figure I NPSR rs32498I T-risk alleles are positively associated with right BLA responsiveness to fear-relevant faces. Left panel: Scatter plot depicting activity of the BLA cluster (middle panel) dependent on genotype. Middle panel: Coronal view $(y=0)$ depicting amygdala responsiveness significantly modulated by genotype (at $p<0.05$, FEW-corrected for the bilateral amygdala). Right panel: Scatter plot depicting a positive correlation $(r=0.37, p=0.004)$ of amygdala cluster responsiveness and TPQ harm avoidance scores.

compare the three genotype groups among each other and for correlation analyses.

For the between-genotype group comparison, we conducted an ANOVA on the cluster contrast values using genotype group as the between-subjects factor. Paralleling the regression analysis, a strong group effect emerged $(\mathrm{F}(1,71)=8.05, p=0.001)$. According to post-hoc Scheffe tests, AT and TT carriers showed significantly increased amygdala responsiveness compared with AA carriers ( $p=0.01$ and $p=0.002$, respectively), whereas no significant difference between AT and TT carriers occurred $(p=0.33)$.

Responsiveness of the BLA cluster correlated significantly with harm avoidance $(r=0.37, p=0.001)$ but not trait anxiety $(r=0.13, p=0.27)$. A multiple regression analysis using BLA responsiveness and NPSR rs324981 T-alleles as predictors of harm avoidance confirmed the strong association of amygdala responsiveness and harm avoidance $(\beta=0.36, p=0.005)$, with no direct effect of T-alleles
( $\beta=0.04, p=0.75)$, indicating an indirect effect on harm avoidance through amygdala responsiveness. Including the interaction term of amygdala responsiveness and NPSR rs324981 T-alleles into the model did not produce any significant results for the interaction term and almost no changes to the other predictors. Furthermore, there was no association of amygdala responsiveness with depression levels as measured using BDI scores $(r=0.09, p=0.46)$.

\section{Effects of Gender}

Given (contradictory) gender-specific results in the categorical association studies with panic disorder (Okamura et al, 2007; Domschke et al, 2010b), we conducted an exploratory correlation of the extracted BLA contrast values and $\mathrm{T}$-alleles within each gender group separately. Our analysis showed significant genotype effects for each gender, with a slightly higher association in women (men: 
$r=0.36, p=0.045$; women: $r=0.49, p=0.001$ ), in line with the female-dominant effects reported in our previous study (Domschke et al, 2010b).

The whole-brain analysis yielded other structures in which neural activity during fear processing was positively associated with NPSR T-alleles (see Table 2). There was no anatomical area showing a significant positive association with number of NPSR A-alleles.

\section{DISCUSSION}

In the present study, we showed a robust effect of a recently described variant in the NPSR gene (NPSR rs324981 A/T) on amygdala responsiveness to fear-relevant facial expressions, with the more active T-allele conferring increased right amygdala responsiveness to fearful/angry faces. This result is entirely in line with previous findings of the more active $\mathrm{T}$-allele to be associated with panic disorder, anxiety sensitivity, and elevated autonomic arousal (Okamura and Reinscheid, 2007; Domschke et al, 2010b; Donner et al, 2010), whereas, quite counter-intuitively, in the rodent model NPS has been shown to act as a potent anxiolytic (see section Introduction). In general, it has to be stated that pharmacological interventions during adulthood do not readily mimic genetically driven alterations during ontogeny. As with the serotonin transporter paradox (see reference Sibille and Lewis, 2006), also for NPS it could be speculated that high NPS levels in early stages of development might have a detrimental effect, whereas at later stages in life NPS might be beneficial with respect to anxiety states. A more specific possible explanation of the apparently contradictive directions of allelic association might be that anxiety is to a great extent conferred through an increased level of arousal (Bouton et al, 2001; Blechert et al, 2007), which in animal models has explicitly been found to be driven by increased NPS activity (Xu et al, 2004; Reinscheid et al, 2005a; Leonard et al, 2008; Rizzi et al, 2008). Increased arousal as conferred by the more active T-allele would furthermore be supported by reports of NPS to cause a significant stimulation of the hypothalamo-pituitaryadrenal (HPA) axis and concomitant increased arousal-like behavior in rats (Smith et al, 2006). Thus, the present finding of association of the more active $\mathrm{T}$-allele with anxiety-relevant brain activation patterns could be due to its arousal-increasing properties as suggested before (cf. Domschke et al, 2010b).

Numerous researchers have argued that the amygdala has a central role in a neural circuit processing fear and anxiety, and mediating arousal (Ledoux, 2000; Davis and Whalen, 2001; Sehlmeyer et al, 2009). High amygdala responsivity to negative stimuli was shown to be associated with cognitive biases favoring processing of negative stimuli (Dannlowski et al, 2007a). The BLA has been argued to represent the central input structure of the amygdaloid complex and is critically involved in the generation of affect (Davis and Whalen, 2001). Also in humans, neuroimaging studies have identified the right BLA as being the main amygdala subarea associated with anxiety or hyperactivity in affective disorders (Etkin et al, 2004; Suslow et al, 2010). Interestingly, in the present study there was no association with depression level and amygdala responsiveness, potentially indicating an anxiety-specific effect and not a general effect of negative emotions. Again, also this is in accordance with animal studies where NPS agonists had no effect on immobility time in a tail suspension test (TST) suggesting no major antidepressant-like activity (Leonard et al, 2008).

Two recent studies have investigated the effects of NPSR rs324981 on brain activation patterns by fMRI: Domschke et al (2010b) reported significantly decreased activity in the anterior cingulate cortex, dorsolateral prefrontal, and orbitofrontal cortex in response to fearful faces in patients with panic disorder carrying the NPSR rs324981 T risk allele, potentially reflecting a distorted cortico-limbic interaction during emotional processing. Using a classic aversive conditioning paradigm in healthy participants, Raczka et al (2010) in turn reported the T-allele to be associated with increased activity in the rostral dorsomedial prefrontal cortex (dmPFC) evoked by the conditioned stimulus, an area that supports the explicit, conscious appraisal of threat stimuli. However, in both studies amygdala modulation by NPSR genotype has not been observed, potentially either due to a small sample size, a partially medicated patient sample, or a ceiling effect of amygdala activation in patient with panic disorder (Domschke et al, $2010 \mathrm{~b}$ ), or the use of a conditioning paradigm (Raczka et al, 2010), which was not specifically designed to activate the amygdala but rather other structures of the fear circuit. By contrast, our present study used a paradigm particularly designed for studying amygdala responsiveness to fearrelevant stimuli.

We detected an association of amygdala responsiveness and NPSR genotype only for the right amygdala. This might be due to the fact that overall there was also a somewhat stronger activation of the right amygdala by this specific task. Furthermore, at a more lenient threshold $(p<0.05$, uncorrected) there was a small cluster showing the same effect also within the left amygdala.

Additionally, we could show that the genetically modulated BLA responsiveness to fear-relevant stimuli was directly associated with the participants' self-reported anxiety-related personality traits (harm avoidance), which is in line with previous neuroimaging studies (Etkin et al, 2004; Fakra et al, 2009; Sehlmeyer et al, 2010; however, see reference Baeken et al, 2010). Albeit we did not find a significant association of trait anxiety with the responsiveness of the investigated BLA cluster, it should be noted that at a more lenient threshold, such as a positive association, was also evident in our sample $(x=34, y=6, z=-20$, $Z=1.89, p_{\text {(uncorrected) }}=0.029$ ).

Our supplemental whole-brain analysis yielded other anatomical areas modulated by NPSR genotype in the same direction as the amygdala. Albeit none of these clusters would survive a rigorous alpha correction for the whole brain, some areas are of particular interest due to their widely reported involvement in emotion processing. Particularly, in line with the findings of Domschke et al, dorsolateral prefrontal cortex clusters (DLPFCs) were observed at the uncorrected significance level, as well as right insular clusters, an orbitofrontal area, and activity within the dorsal ACC. The OFC has a role in the mediation of autonomic changes accompanying affective states produced in response to emotive stimuli or contexts, suggestive of a role for this region in the automatic regulation of emotional 
behavior (Phillips et al, 2003). The association of neural responses in these areas with $\mathrm{T}$-alleles might indicate an increased subjective experience of emotions and autonomic arousal during the processing of the angry and fearful expressions. On the other hand, the DLPFC and dorsal ACC were shown to be involved in executive control and emotion regulation. This might reflect a compensatory engagement in (healthy) risk allele carriers who experience the necessity to regulate their emotions more strongly than subjects with lower amygdala responsiveness.

Some limitations must be acknowledged. We have not genotyped our subjects for other potentially relevant polymorphisms, which could modulate amygdala responsiveness, for example, 5-HTTLPR, 5- $\mathrm{HT}_{1 \mathrm{~A}} 1019 \mathrm{C} / \mathrm{G}$, or neuropeptide-Y (NPY) among others (Dannlowski et al, 2007b, 2008, 2009, 2010; Domschke et al, 2010a). The present effect size of $r=0.44$ (equivalent to $d=0.99$ ) is in the same range as the reported effects of neuropeptide-Y (Domschke et al, 2010a) and potentially even higher compared with 5-HTTLPR (Munafò et al, 2008). However, a direct comparison of different polymorphisms regarding effect sizes is not possible with our present data. The sample size was relatively small for a genetic association study, but on the other hand it was large for an imaging genetics study, exceeding recommended sample sizes for this kind of analysis (Munafò et al, 2008). Nonetheless, our sample size was particularly small for analyzing subsamples stratified for gender and therefore these results should be treated with care.

In sum, our study provides further support of a strong role of NPS and NPSR in the genetic and neural underpinnings of anxiety and anxiety disorders. It provides a missing link between the results of animal studies, human genetic association studies, and neuroimaging results, and integrates these findings. Taken together, these findings might stimulate future studies involving the exploration of therapeutic agents targeting the NPS system in anxiety disorders.

\section{ACKNOWLEDGEMENTS}

We thank Ahmad Hariri for providing the fMRI paradigm. We further thank Mrs Nina Nagelmann and Mrs Kathrin Schwarte for skillful technical support during the fMRI sessions and genotyping, respectively. The study was supported by grants from the Deutsche Forschungsgemeinschaft (DFG; SFB-TRR-58 project C2 to KD and C1 to PZ), Innovative Medizinische Forschung (IMF DA120309 and IMF DA211012 to UD), and Rolf-Dierichs-Stiftung (ZUW80037 to UD).

\section{DISCLOSURE}

Professor Volker Arolt, MD, PhD, is member of advisory boards and/or gave presentations for the following companies: Astra-Zeneca, Janssen-Organon, Lilly, Lundbeck, Servier, Pfizer, and Wyeth. He chairs the committee for the 'Wyeth Research Award Depression and Anxiety', now the DGPPN/Pfizer 'Clinical Neuroscience Award'. Professor Katharina Domschke, MA, MD, PhD, is on the speakers' board of Pfizer, Lilly, and Bristol-Myers Squibb, and has received funding from Astra-Zeneca. These affiliations have no relevance to the work covered in the paper. All other authors have no conflicts of interest to declare, financial or otherwise.

\section{REFERENCES}

American Psychiatric Association (1994). Diagnostic and Statistical Manual of Mental Disorders, 4th ed. American Psychiatric Association: Washington, DC.

Amunts K, Kedo O, Kindler M, Pieperhoff P, Mohlberg H, Shah NJ et al (2005). Cytoarchitectonic mapping of the human amygdala, hippocampal region and entorhinal cortex: intersubject variability and probability maps. Anat Embryol 210: 343-352.

Baeken C, Van Schuerbeek P, De Raedt R, Bossuyt A, Vanderhasselt M-A, De Mey J et al (2010). Passively viewing negatively valenced baby faces attenuates left amygdala activity in healthy females scoring high on 'Harm Avoidance'. Neurosci Lett 478: 97-101.

Beck AT, Steer RA (1987). Beck Depression Inventory: Manual. Psychological Corporation Harcourt Brace Jovanovich: San Antonio, TX.

Bernier V, Stocco R, Bogusky MJ, Joyce JG, Parachoniak C, Grenier K et al (2006). Structure-function relationships in the neuropeptide $S$ receptor: molecular consequences of the asthma-associated mutation N107I. J Biol Chem 281: 24704-24712.

Blechert J, Michael T, Grossman P, Lajtman M, Wilhelm FH (2007). Autonomic and respiratory characteristics of posttraumatic stress disorder and panic disorder. Psychosom Med 69: 935-943.

Bouton ME, Mineka S, Barlow DH (2001). A modern learning theory perspective on the etiology of panic disorder. Psychol Rev 108: 4-32.

Cloninger CR (1987). A systematic method for clinical description and classification of personality variants. A proposal. Arch Gen Psychiatry 44: 573-588.

Dannlowski U, Konrad C, Kugel H, Zwitserlood P, Domschke K, Schöning $S$ et al (2010). Emotion specific modulation of automatic amygdala responses by 5-HTTLPR genotype. NeuroImage 53: 893-898.

Dannlowski U, Ohrmann P, Bauer J, Deckert J, Hohoff C, Kugel H et al (2008). 5-HTTLPR biases amygdala activity in response to masked facial expressions in major depression. Neuropsychopharmacology 33: 418-424.

Dannlowski U, Ohrmann P, Bauer J, Kugel H, Arolt V, Heindel W et al (2007a). Amygdala reactivity to masked negative faces is associated with automatic judgmental bias in major depression: a 3 T fMRI study. J Psychiatry Neurosci 32: 423-429.

Dannlowski U, Ohrmann P, Bauer J, Kugel H, Baune BT, Hohoff C et al (2007b). Serotonergic genes modulate amygdala activity in major depression. Genes Brain Behav 6: 672-676.

Dannlowski U, Ohrmann P, Konrad C, Domschke K, Bauer J, Kugel $\mathrm{H}$ et al (2009). Reduced amygdala-prefrontal coupling in major depression: association with MAOA genotype and illness severity. Int J Neuropsychopharmacol 12: 11-22.

Davis M, Whalen PJ (2001). The amygdala: vigilance and emotion. Mol Psychiatry 6: 13-34.

Domschke K, Dannlowski U (2010). Imaging genetics of anxiety disorders. NeuroImage 53: 822-831.

Domschke K, Dannlowski U, Hohoff C, Ohrmann P, Bauer J, Kugel H et al (2010a). Neuropeptide Y (NPY) gene: impact on emotional processing and treatment response in anxious depression. Eur Neuropsychopharmacol 20: 301-309.

Domschke K, Reif A, Weber H, Richter J, Hohoff C, Ohrmann P et al (2010b). Neuropeptide $S$ receptor gene-converging evidence for a role in panic disorder. Mol Psychiatry (in press).

Donner J, Haapakoski R, Ezer S, Melén E, Pirkola S, Gratacòs M et al (2010). Assessment of the neuropeptide $S$ system in anxiety disorders. Biol Psychiatry 68: 474-483. 
Eickhoff SB, Stephan KE, Mohlberg H, Grefkes C, Fink GR, Amunts $\mathrm{K}$ et al (2005). A new SPM toolbox for combining probabilistic cytoarchitectonic maps and functional imaging data. NeuroImage 25: 1325-1335.

Ekman P, Friesen WV (1976). Pictures of Facial Affect. Consulting Psychologists Press: Palo Alto.

Etkin A, Klemenhagen KC, Dudman JT, Rogan MT, Hen R, Kandel ER et al (2004). Individual differences in trait anxiety predict the response of the basolateral amygdala to unconsciously processed fearful faces. Neuron 44: 1043-1055.

Etkin A, Wager TD (2007). Functional neuroimaging of anxiety: a meta-analysis of emotional processing in PTSD, social anxiety disorder, and specific phobia. Am J Psychiatry 164: 1476-1488.

Fakra E, Hyde LW, Gorka A, Fisher PM, Muñoz KE, Kimak M et al (2009). Effects of HTR1A C (-1019)G on amygdala reactivity and trait anxiety. Arch Gen Psychiatry 66: 33-40.

Fendt M, Imobersteg S, Bürki H, McAllister KH, Sailer AW (2010). Intra-amygdala injections of neuropeptide $S$ block fearpotentiated startle. Neurosci Lett 474: 154-157.

Hariri AR, Drabant EM, Muñoz KE, Kolachana BS, Mattay VS, Egan MF et al (2005). A susceptibility gene for affective disorders and the response of the human amygdala. Arch Gen Psychiatry 62: $146-152$.

Hariri AR, Mattay VS, Kolachana BS, Tessitore A, Fera F, Goldman D et al (2002). Serotonin transporter genetic variation and the response of the human amygdala. Science 297: 400-403.

Hettema JM, Neale MC, Kendler KS (2001). A review and metaanalysis of the genetic epidemiology of anxiety disorders. Am J Psychiatry 158: 1568-1578.

Jüngling K, Seidenbecher T, Sosulina L, Lesting J, Sangha S, Clark $\mathrm{SD}$ et al (2008). Neuropeptide S-mediated control of fear expression and extinction: role of intercalated GABAergic neurons in the amygdala. Neuron 59: 298-310.

Lancaster JL, Woldorff MG, Parsons LM, Liotti M, Freitas CS, Rainey L et al (2000). Automated Talairach atlas labels for functional brain mapping. Hum Brain Mapp 10: 120-131.

Laux L, Glanzmann P, Schaffner P, Spielberger CD (1981). Das State-Trait Angstinventar. Beltz: Weinheim, Germany.

Ledoux JE (2000). Emotion circuits in the brain. Annu Rev Neurosci 23: 155-184.

Lehrl S (1995). Mehrfachwahl-Wortschatz-Intelligenztest MWT-B. Hogrefe: Göttingen, Germany.

Leonard SK, Dwyer JM, Sukoff Rizzo SJ, Platt B, Logue SF, Neal SJ et al (2008). Pharmacology of neuropeptide $S$ in mice: therapeutic relevance to anxiety disorders. Psychopharmacology 197: 601-611.

Maldjian JA, Laurienti PJ, Kraft RA, Burdette JH (2003). An automated method for neuroanatomic and cytoarchitectonic atlasbased interrogation of fMRI data sets. NeuroImage 19: 1233-1239.

Meis S, Bergado-Acosta JR, Yanagawa Y, Obata K, Stork O, Munsch T (2008). Identification of a neuropeptide $S$ responsive circuitry shaping amygdala activity via the endopiriform nucleus. PLoS One 3: e2695.

Meyer-Lindenberg A, Buckholtz JW, Kolachana BS, Hariri A, Pezawas L, Blasi G et al (2006). Neural mechanisms of genetic risk for impulsivity and violence in humans. Proc Natl Acad Sci USA 103: 6269-6274.

Munafò MR, Brown SM, Hariri AR (2008). Serotonin transporter (5-HTTLPR) genotype and amygdala activation: a meta-analysis. Biol Psychiatry 63: 852-857.

Okamura N, Hashimoto K, Iyo M, Shimizu E, Dempfle A, Friedel S et al (2007). Gender-specific association of a functional coding polymorphism in the neuropeptide $S$ receptor gene with panic disorder but not with schizophrenia or attentiondeficit/hyperactivity disorder. Prog Neuropsychopharmacol Biol Psychiatry 31: 1444-1448.

Okamura N, Reinscheid RK (2007). Neuropeptide S: a novel modulator of stress and arousal. Stress 10: 221-226.

Pape H-C, Jüngling K, Seidenbecher T, Lesting J, Reinscheid RK (2010). Neuropeptide S: a transmitter system in the brain regulating fear and anxiety. Neuropharmacology 58: 29-34.

Pezawas L, Meyer-Lindenberg A, Drabant EM, Verchinski BA, Muñoz KE, Kolachana BS et al (2005). 5-HTTLPR polymorphism impacts human cingulate-amygdala interactions: a genetic susceptibility mechanism for depression. Nat Neurosci $\mathbf{8}$ : 828-834.

Phillips ML, Drevets WC, Rauch SL, Lane R (2003). Neurobiology of emotion perception I: the neural basis of normal emotion perception. Biol Psychiatry 54: 504-514.

Raczka K, Gartmann N, Mechias M-L, Reif A, Büchel C, Deckert J et al (2010). A neuropeptide $S$ receptor variant associated with overinterpretation of fear reactions: a potential neurogenetic basis for catastrophizing. Mol Psychiatry 15: 1067-1074.

Reinscheid RK, Xu Y-L, Civelli O (2005a). Neuropeptide S: a new player in the modulation of arousal and anxiety. Mol Interv 5: $42-46$.

Reinscheid RK, Xu Y-L, Okamura N, Zeng J, Chung S, Pai R et al (2005b). Pharmacological characterization of human and murine neuropeptide s receptor variants. J Pharmacol Exp Ther 315: 1338-1345.

Rizzi A, Vergura R, Marzola G, Ruzza C, Guerrini R, Salvadori S et al (2008). Neuropeptide $S$ is a stimulatory anxiolytic agent: a behavioural study in mice. Br J Pharmacol 154: 471-479.

Sehlmeyer C, Dannlowski U, Schöning S, Kugel H, Pyka M, Pfleiderer B et al (2010). Neural correlates of trait anxiety in fear extinction. Psychol Med 41: 789-798.

Sehlmeyer C, Schöning S, Zwitserlood P, Pfleiderer B, Kircher T, Arolt $\mathrm{V}$ et al (2009). Human fear conditioning and extinction in neuroimaging: a systematic review. PLoS One 4: e5865.

Sibille E, Lewis DA (2006). SERT-ainly involved in depression, but when? Am J Psychiatry 163: 8-11.

Smith KL, Patterson M, Dhillo WS, Patel SR, Semjonous NM, Gardiner JV et al (2006). Neuropeptide S stimulates the hypothalamo-pituitary-adrenal axis and inhibits food intake. Endocrinology 147: 3510-3518.

Suslow T, Konrad C, Kugel H, Rumstadt D, Zwitserlood P, Schöning $S$ et al (2010). Automatic mood-congruent amygdala responses to masked facial expressions in major depression. Biol Psychiatry 67: 155-160.

Tzourio-Mazoyer N, Landeau B, Papathanassiou D, Crivello F, Etard O, Delcroix N et al (2002). Automated anatomical labeling of activations in SPM using a macroscopic anatomical parcellation of the MNI MRI single-subject brain. NeuroImage 15: 273-289.

Vitale G, Filaferro M, Ruggieri V, Pennella S, Frigeri C, Rizzi A et al (2008). Anxiolytic-like effect of neuropeptide $S$ in the rat defensive burying. Peptides 29: 2286-2291.

Wittchen H-U, Wunderlich U, Gruschwitz S, Zaudig M (1997). SKID-I. Strukturiertes Klinisches Interview für DSM-IV. Hogrefe: Göttingen, Germany.

Xu Y-L, Reinscheid RK, Huitron-Resendiz S, Clark SD, Wang Z, Lin SH et al (2004). Neuropeptide S: a neuropeptide promoting arousal and anxiolytic-like effects. Neuron 43: 487-497.

Zhou Z, Zhu G, Hariri AR, Enoch MA, Scott D, Sinha R et al (2008). Genetic variation in human NPY expression affects stress response and emotion. Nature 452: 997-1001. 